\title{
Sea Ice Warning Visualization and Path Planning for Ice Navigation Based on Radar Image Recognition
}

\author{
Tsung-Hsuan Hsieh \\ Merchant Marine College, Shanghai Maritime University, Shanghai, China, zxxie@shmtu.edu.cn \\ Shengzheng Wang \\ Merchant Marine College, Shanghai Maritime University, Shanghai, China \\ Huijia Gong \\ Merchant Marine College, Shanghai Maritime University, Shanghai, China \\ Wei Liu \\ Merchant Marine College, Shanghai Maritime University, Shanghai, China \\ Ning Xu \\ Polar Research Institute of China, Shanghai, China
}

Follow this and additional works at: https://jmstt.ntou.edu.tw/journal

Part of the Fresh Water Studies Commons, Marine Biology Commons, Ocean Engineering Commons, Oceanography Commons, and the Other Oceanography and Atmospheric Sciences and Meteorology Commons

\section{Recommended Citation}

Hsieh, Tsung-Hsuan; Wang, Shengzheng; Gong, Huijia; Liu, Wei; and Xu, Ning (2021) "Sea Ice Warning Visualization and Path Planning for Ice Navigation Based on Radar Image Recognition," Journal of Marine Science and Technology. Vol. 29: Iss. 3, Article 4.

DOI: $10.51400 / 2709-6998.1433$

Available at: https://jmstt.ntou.edu.tw/journal/vol29/iss3/4

This Research Article is brought to you for free and open access by Journal of Marine Science and Technology. It has been accepted for inclusion in Journal of Marine Science and Technology by an authorized editor of Journal of Marine Science and Technology. 


\title{
Sea Ice Warning Visualization and Path Planning for Ice Navigation Based on Radar Image Recognition
}

\author{
Tsung-Hsuan Hsieh ${ }^{a}{ }^{a}$, Shengzheng Wang ${ }^{a}$, Huijia Gong ${ }^{a}$, Wei Liu ${ }^{a}$, Ning $\mathrm{Xu}^{\mathrm{b}}$ \\ ${ }^{a}$ Merchant Marine College, Shanghai Maritime University, Shanghai, China \\ ${ }^{\mathrm{b}}$ Polar Research Institute of China, Shanghai, China
}

\begin{abstract}
Ice navigation is currently one of the important issues in path planning studies. Most studies are using sea ice models or ice charts for large-scale path planning, but the weather and ice conditions in the polar waters usually change quickly and are not easy to predict. Under these conditions, small-scale path planning that can be adjusted in time for sea ice collision avoidance is needed. This study uses marine radar imaging to reconstruct the ice navigation scene, and establishes a visualization function of sea ice warning on the radar image. We combine the bidirectional rapidly-exploring random tree (B-RRT) algorithm and the greedy algorithm to propose a path planning algorithm that generates various path planning schemes for ice navigation. After a number of path planning schemes are quickly generated, the path risk index proposed in this study can be used to evaluate and choose a relatively optimal path planning scheme with lower risk, as well as serve as an assistant reference for path planning decisions in ice navigation.
\end{abstract}

Keywords: Ice navigation, Path planning, Radar image, Risk index

\section{Introduction}

I $\mathrm{n}$ recent years, the polar regions have become an international development hotspot. The Arctic area has shorter shipping routes and a wealth of oil, gas and mineral resources, and, in addition to scientific research, tourism activities in the Antarctic area have also increased year by year. As the demand for navigation in the polar waters increases continually, route optimization and sea ice collision avoidance have increasingly attracted the interest of researchers.

For ice navigation, in addition to the use of searchlights, telescopes and other traditional equipment to detect ice conditions, marine radar is the second pair of eyes for navigators and an important ice detection tool. The International Code for Ships Operating in Polar Waters (Polar Code) clearly states that radar equipped with enhanced ice detection capability should be promoted [7]. The Guidelines for Polar Water Operational Manual published by the China Classification Society also mentions that, although the ability of radar to detect of ice floes with different characteristics has some limitations under different sea and weather conditions, it is still the most effective equipment for ice detection at present [1]. Even though the thickness and structure of the sea ice cannot be known from ordinary marine radars, real-time sea ice coverage conditions around the ship can still be obtained.

Compared with widely studied ship path planning, the problem of path planning for ice navigation has only recently drawn concern. Based on various sources of sea ice data, scholars have used different algorithms to solve navigation paths in different regions. Most of the data sources for these studies are sea ice models $[12,2,19,5,3,20,11,18,15]$ or ice charts $[4,17]$, and the navigation areas are mostly in large-scale spaces, such as the Arctic Ocean 
$[2,19,3]$, the Baltic Sea $[12,5,18,15]$, the Norwegian Sea [20], the Barents Sea [11], the Canadian Arctic [17], and the Gulf of St. Lawrence [4]. In addition, most studies used algorithms that can quickly obtain the solutions, such as the $\mathrm{A}^{*}$ algorithm $[5,3,17,15]$, the Dijkstra algorithm [19], Powell's method [12] and so on. [16] have done a detailed collation and comparison of these studies.

Different from the large-scale path planning studies, this study uses radar images as the source of sea ice data, which belong to the small-scale space problem of path planning for sea ice collision avoidance. Most ships navigating in the polar waters have certain anti-ice capability, however, the closer the ship is to sea ice, the higher the risk of accidents, such as getting stuck, and suffering various degrees of ice damage. Therefore, a ship should still maintain a certain distance from the sea ice as much as possible. Due to the smaller display range of radar image, the computing time requirements of the algorithm is shorter. At present, there have been few studies on path planning for ice navigation using radar images, Takagi et al. [21] is one exception, however, unlike their study, this study not only considers the distance between the sea ice and the ship, but also considers the area of sea ice. This study used the bidirectional rapidlyexploring random tree (B-RRT) algorithm and the greedy algorithm to plan the path, and the running time of path generation was recorded and compared in the subsequent demonstration examples.

With the support from the Polar Research Institute of China, this study collected the radar playback video of the polar research vessel Xuelong during China's 33rd Antarctic expedition, from Shanghai to Zhongshan Station for 28 days (November 2 to 29, 2016). The Xuelong approached $60^{\circ}$ south latitude on November 27, 2016, where it encountered obvious sea ice in the surrounding waters. The subsequent examples used in this study are from the radar image of that day. In this study, path planning is based on the static timing point, without considering the dynamic tracking and clutter interference.

\section{Reconstruction of the ice navigation scene}

Based on image recognition technology, this study reconstructs the ice navigation scene through five steps, including radar image reading, key information acquisition, circular display area capture, ownship position and short-term target position confirmation, and unknown obstacle area extraction. These steps are specified as follows:

\subsection{Radar image reading}

According to the set frames rate (i.e., frames per second, FPS), radar images at specific time points can be captured from radar video. This study collected the radar playback video of the polar research vessel Xuelong during its Antarctic journey in 2016, and used image software to capture the radar image at 6:53 on November 27, 2016, as shown in Fig. 1. The resolution of captured radar image was $1280 \times 1024$ pixels, which was then converted into a high-dimensional pixel matrix, containing the RGB color values assigned at all pixel positions of the image.

\subsection{Key information acquisition}

According to the requirements of the Revised Performance Standards for Radar Equipment [6], the information that can obtained from the radar image includes: display mode (including true motion mode and relative motion mode), display orientation (including North-up, Course-up, Headup, own-ship position), the selected range scale, the range ring interval, heading, speed toward water, course over ground, speed over ground, and planning path.

\subsection{Circular display area capture}

The circular display area of radar image is the region of interest (ROI), which is the main display area of the ice navigation scene. In order to filter out the redundant information of the other areas, a mask region matrix is used to represent the ROI. The values inside the ROI are set to 1 , and the pixel values outside the ROI are set to 0 . By multiplying the high-dimensional pixel matrix of the original image by the mask area matrix, the image of a circular display area can be captured, as shown in Fig. 2.

\subsection{Own-ship position and short-term target position confirmation}

As shown in Fig. 2, even if the image display mode is off center on the screen, the own-ship position is still a consistent common reference point (CCRP) and the center of the range rings. The short-term target position can be judged and known from the heading, and the intersection of the planning path and the bearing scale. The definitions and specifications of the appearance, color, and symbol of the planning path are found in the Guidelines for the Presentation of Navigation-related Symbols, Terms 


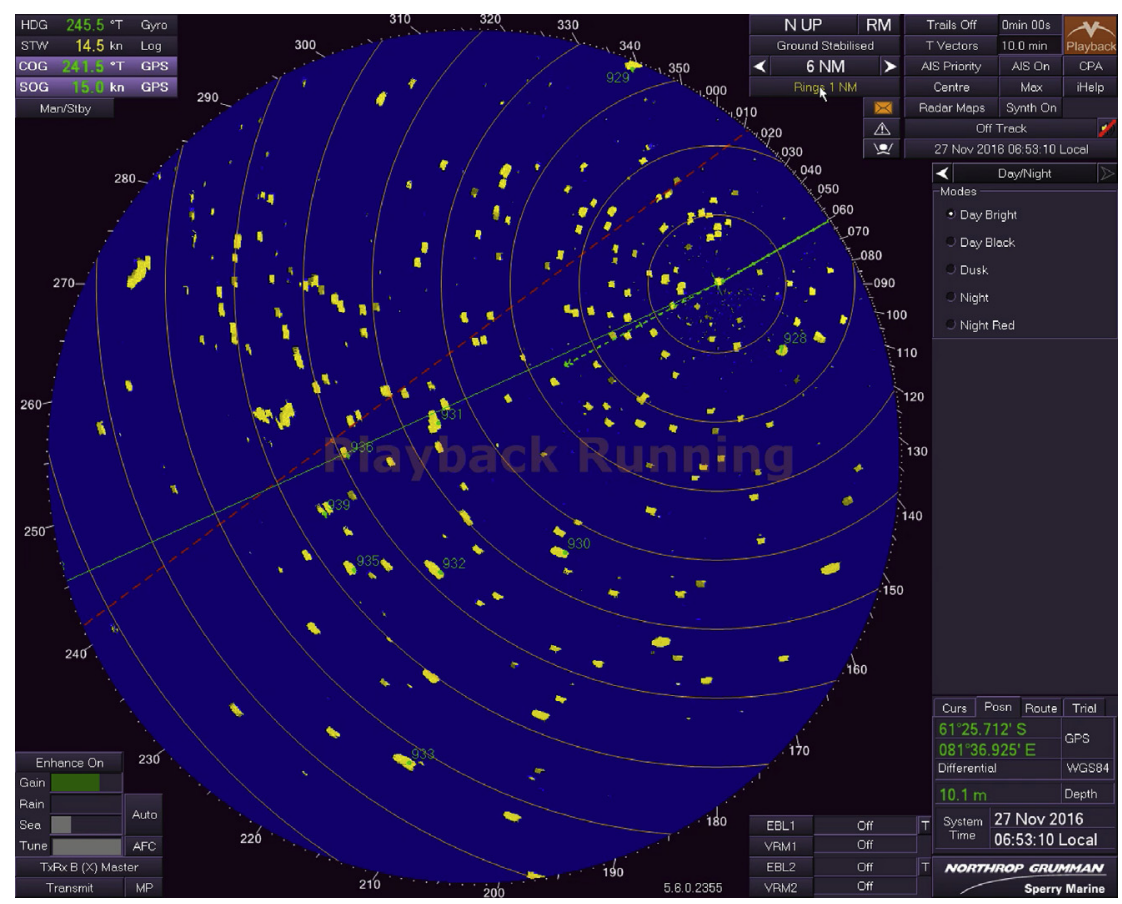

Fig. 1. The original radar image captured from radar playback video.

and Abbreviations [8]. Accordingly, the image software can read the coordinates of own-ship position and short-term target position on the radar image.

\subsection{Unknown obstacle area extraction}

When a ship enters the Arctic area at $60^{\circ}$ north latitude or the Antarctic area at $60^{\circ}$ south latitude, there is a high probability that the unknown obstacle areas displayed in the circular display area are sea ice. In order to extract all unknown obstacle areas, as shown in Fig. 3, the unknown obstacle areas are set where the pixel colors are close to yellow, to 255 (which are displayed in white), and the other areas are set to 0 (which are displayed in black). From this, the binary image of all unknown

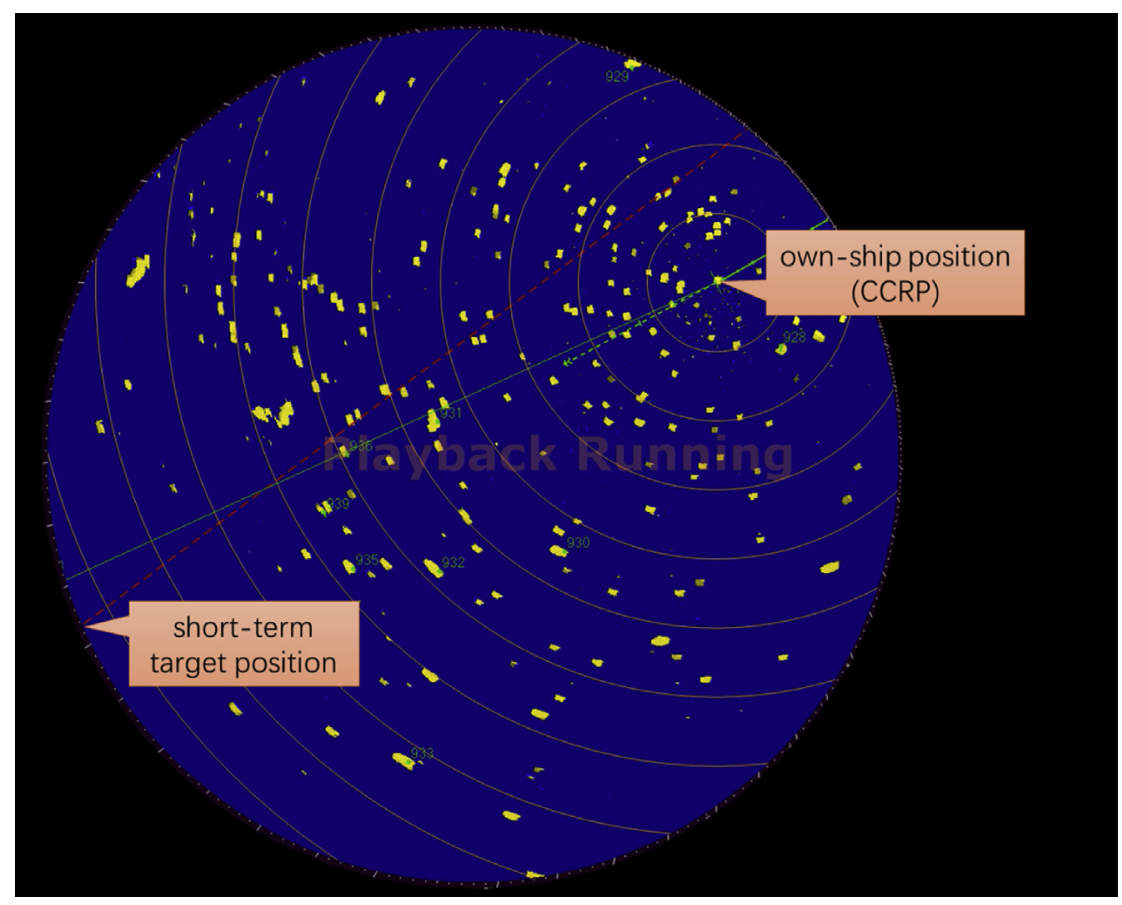

Fig. 2. The circular display area image captured from original radar image. 


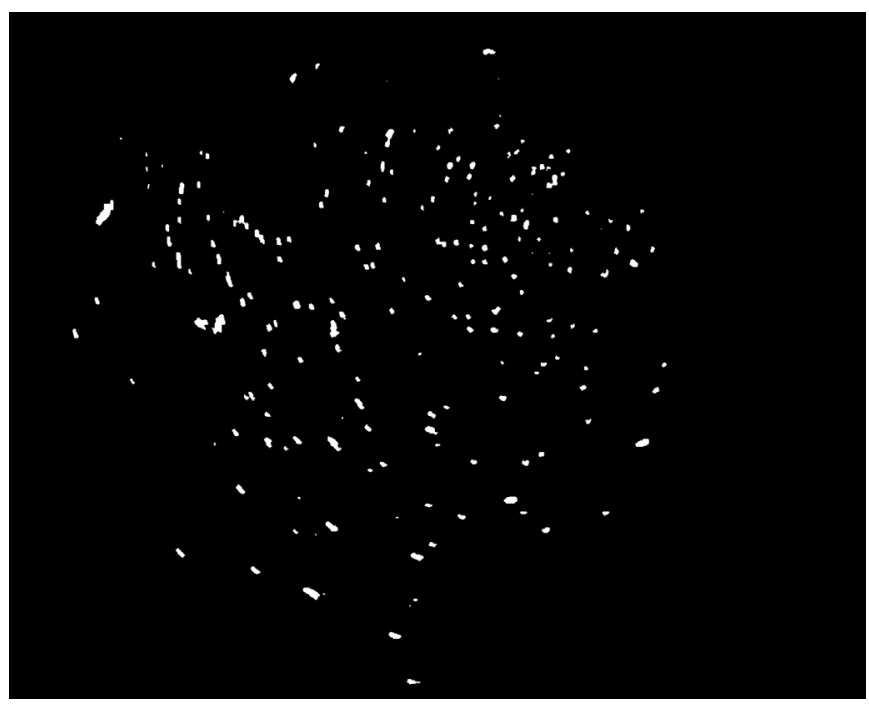

Fig. 3. The binary image extracted from the circular display area image.

obstacle areas can be extracted. Finally, an image filtering algorithm can be used to remove noise from the image and make the unknown obstacle areas smoother. The erosion and dilation methods of the image processing technique can also be used to adjust the size of the unknown obstacle areas to reduce the phenomenon of the unknown obstacle areas becoming smaller due to the image filtering algorithm.

After these five steps, the own-ship position, short-term target position and unknown obstacle areas can be recognized from the radar image, and the ice navigation scene can be reconstructed. This lays a foundation for the sea ice warning visualization such as the one we constructed in Chapter 3 , and the path planning for ice navigation in Chapter 4.

\section{Establishment of sea ice warning visualization}

In the appendix to the Guidelines on Methodologies for Assessing Operational Capabilities and Limitations in Ice [9] can be found the Polar Operational Limit Assessment Risk Indexing System (POLARIS), providing a risk index outcome (RIO) to assess the limitation for operation in ice. However, the RIO cannot identify the risk degree of each unknown obstacle area displayed on the radar image. Therefore, this study proposes a radar sea ice risk index (RI) to identify the risk degree of each sea ice area to the ship. Based on this risk index, we can establish a visualization function of sea ice warning on the radar image to assist the ice navigation.
The specific instructions are as follows:

Through the boundary tracing method of the image processing technique, the exterior boundaries and label of each unknown obstacle area in the ice navigation scene, reconstructed in Chapter 2, can be obtained. However, the exterior boundaries and labels of the unknown obstacle area, overlapping with the CCRP, should be eliminated, because this unknown obstacle is likely to be the ship itself. The surrounding sea ice cover centered on own-ship position can then be obtained from the ordinary marine radar image.

Accordingly, this study creates a radar sea ice risk index (RI) by taking the area of sea ice and the distance between the sea ice and the ship as variables, as shown in Eq. (1):

$R I_{i}=\frac{A_{i}}{D_{i}}$

in which $R I_{i}$ is the risk index of the $i$ th sea ice on the radar image; $A_{i}$ is the area of the $i$ th sea ice, in pixels; $D_{i}$ is the distance between the $i$ th sea ice and the ship, i.e. the distance between the centroid of the $i$ th sea ice and the CCRP, in pixels.

The area of sea ice is directly proportional to the risk degree of the sea ice; the larger the area of sea ice, the higher the value of the RI. The distance between the sea ice and the ship is inversely proportional to the risk degree of the sea ice; the closer the distance between the sea ice and the ship, the higher the value of the RI. The RI represents the relative risk degree of a given sea ice area; the 


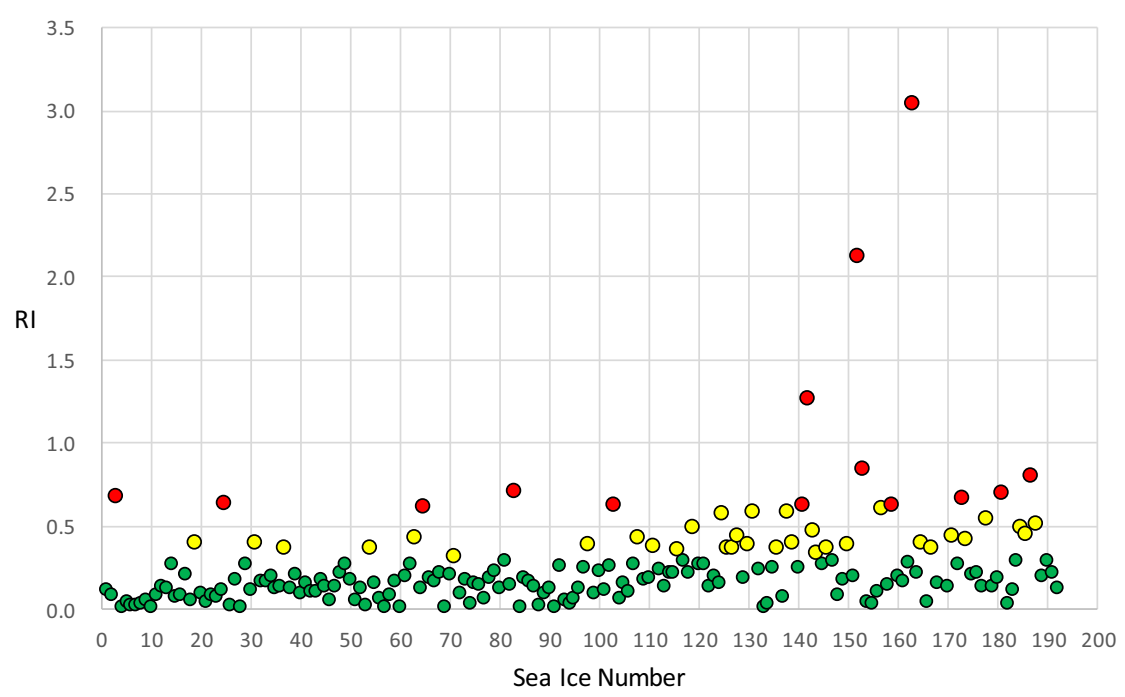

Fig. 4. Schematic diagram for the distribution of RI values.

higher the value of the RI, the higher the risk degree of a given sea ice area. The thickness and structure of sea ice cannot be known from the ordinary marine radar image, and the small areas of sea ice may also be thick icebergs, but the sea ice with large areas and in close proximity must be given special attention and precautions taken during navigation.

In this study, sea ice areas with an RI value less than 0.3 are defined as low-risk areas. Sea ice areas with an RI value greater than or equal to 0.3 and less than 0.6 are defined as medium-risk areas, and sea ice areas with an RI value greater than or equal to 0.6 are defined as high-risk areas. Taking the ice

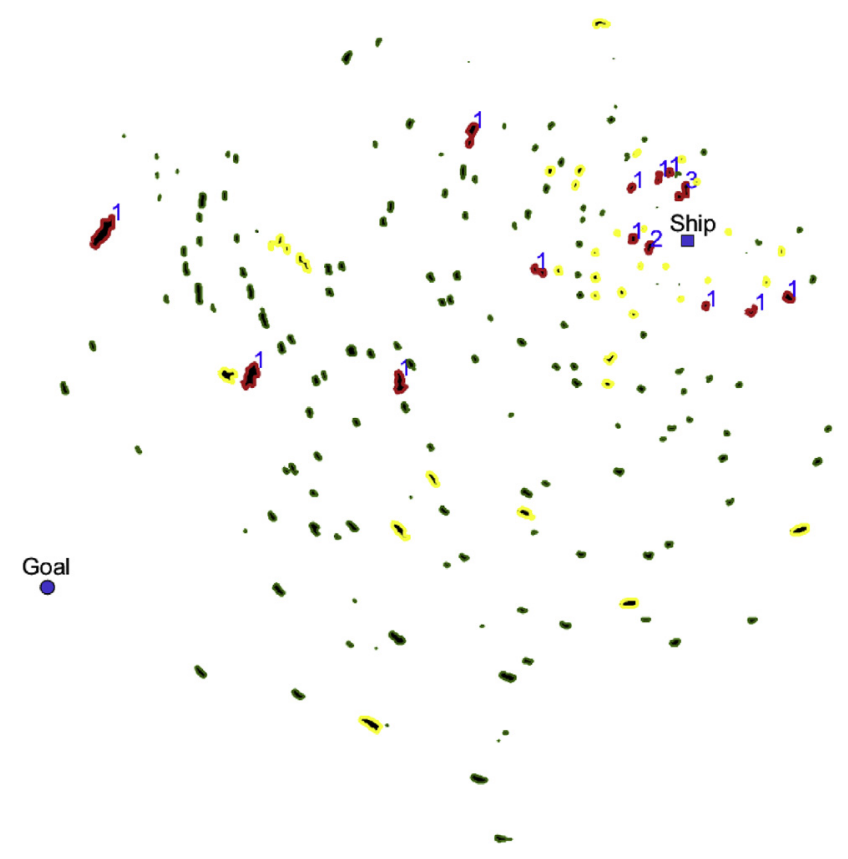

Fig. 5. The visualization function of sea ice warning on radar image. navigation scene reconstructed in Chapter 2 as an example, 192 sea ice areas were marked after image recognition and processing, including 145 low-risk areas, 33 medium-risk areas and 14 high-risk areas. The distribution of RI values is shown in Fig. 4. The low-risk areas are shown in green, medium-risk areas are shown in yellow, and high-risk areas are shown in red.

Based on the RI, a visualization function of sea ice warning is established on the radar image for ice navigation. Taking the ice navigation scene reconstructed in Chapter 2 as an example, as shown in Fig. 5, the own-ship position is displayed in a blue square, the short-term target position is displayed in a blue circle, the boundaries of low-risk sea ice areas $\left(R I_{i}<0.3\right)$ are displayed in green; the boundaries of medium-risk sea ice areas $\left(0.3 \leq R I_{i}<0.6\right)$ are displayed in yellow; the boundaries of high-risk sea ice areas $\left(R I_{i} \geq 0.6\right)$ are displayed in red, and their RI values are rounded to integers and marked in blue numerics. From this, the relatively high-risk sea ice areas can be quickly identified from the boundary colors and marked numeric values of the sea ice areas.

\section{Generation and evaluation of path planning for ice navigation}

Through the five steps based on image recognition technology used in Chapter 2, and the radar sea ice risk index (RI) proposed in Chapter 3, the ice navigation scene can be reconstructed and the risk degree of each sea ice area to the ship can be identified. Within the scope of this ice navigation scene, the own-ship position can be regarded as the starting point, the short-term target position on the 
planning path can be regarded as the end point, and the unknown obstacle areas can be regarded as sea ice areas. By using the proposed path planning algorithms, various path planning schemes can be generated. According to the proposed risk assessment index, a relatively optimal path planning scheme can be chosen. The specific instructions are as follows:

\subsection{Generation of path planning for ice navigation}

In this study, the bidirectional rapidly-exploring random tree (B-RRT) algorithm and the greedy algorithm are used for path planning for ice navigation.

The rapidly-exploring random tree (RRT) algorithm was proposed by [13]; and its basic idea is shown in Fig. 6. At first, a point is generated randomly in configuration space, i.e., the random vertex $\left(q_{\text {rand }}\right)$, and the probability of the end point $\left(q_{\text {goal }}\right)$ being used as a random vertex is set to 0.5 . Within all vertices, find the vertex closest to the random vertex, i.e., the nearest vertex $\left(q_{\text {near }}\right)$, and the starting point $\left(q_{\text {init }}\right)$ is the nearest vertex at the beginning, then use the collision detection function to judge whether there are any obstacles on the connection line between the nearest vertex $\left(q_{\text {near }}\right)$ and the random vertex $\left(q_{\text {rand }}\right)$. If there are obstacles, regenerate another random vertex $\left(q_{\text {rand }}\right)$ and carry out collision detection again until there are no obstacles. If there are no obstacles, use a preset step size $(\Delta q)$ to add a vertex in the direction of the nearest vertex to the random vertex, i.e., the new vertex $\left(q_{\text {new }}\right)$. Accordingly, a vertex and an edge length will be added in the RRT, a new random vertex $\left(q_{\text {rand }}\right)$ will be regenerated, and the operation will be repeated until the distance between the new vertex $\left(q_{\text {new }}\right)$ and the end point $\left(q_{\text {goal }}\right)$ is less than the set threshold value; and there the operation ends.

Based on the RRT algorithm, the B-RRT algorithm adds new vertices to the configuration space from the starting point and the end point simultaneously, which enables the path to be generated more quickly $[13,14,10]$. However, by using a preset step

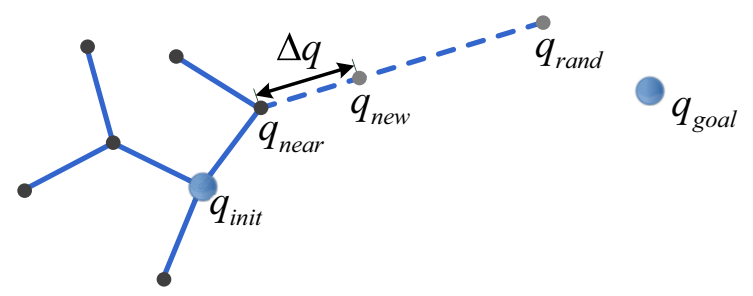

Fig. 6. Schematic diagram for the basic idea of RRT algorithm.

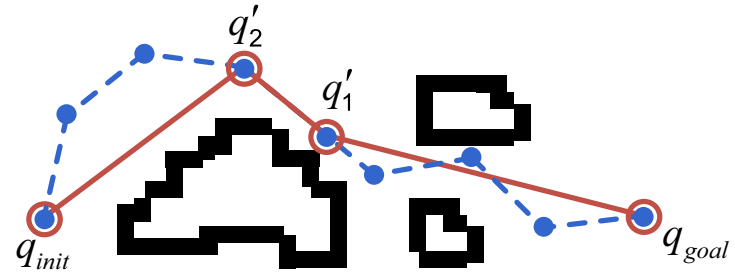

Fig. 7. Schematic diagram for the basic idea of greedy algorithm.

size $(\Delta q)$ to add new vertices, the RRT and B-RRT algorithms are prone to generate redundant vertices. To improve on this shortcoming, this study also combines the greedy algorithm (Fig. 7). The original path generated by the B-RRT algorithm is shown as a blue dashed line. From the starting point $\left(q_{\text {init }}\right)$, carry out the collision detection of each vertex on the original path sequentially, i.e., judge in sequence whether there are any obstacles on the connection line between each vertex and the end point $\left(q_{\text {goal }}\right)$. The vertex is then set so that it can pass the collision detection earliest as the first vertex of the new path $\left(q_{1}{ }^{\prime}\right)$. Then, take this vertex as the new end point of detection (i.e., instead of the $q_{\text {goal }}$ ). From the starting point $\left(q_{\text {init }}\right)$, again carry out the collision detection of each vertex on the path of the starting point $\left(q_{\text {init }}\right)$ to a new end point of detection in sequence, and set the vertex so that it can pass the collision detection earliest as the second vertex of the new path $\left(q_{2}{ }^{\prime}\right)$. Next, take this vertex as the new end point of detection (i.e., instead of the $q_{1}{ }^{\prime}$ ). The operation is repeated until there are no obstacles on the connection line between the starting point $\left(q_{\text {init }}\right)$ and the vertex, which is last set on the new path. The new path generated by combining the use of the greedy algorithm is shown in Fig. 7 as a solid red line.

Taking the ice navigation scene reconstructed in Chapter 2 as an example, a sea ice collision avoidance path planning scheme is randomly generated by using the B-RRT algorithm. The step size is set to 50 pixels, and the threshold value is also set to 50 pixels, as shown in Fig. 8. The B-RRT algorithm adds new vertices to the configuration space (i.e., the reconstructed ice navigation scene) from the starting point (i.e., the own-ship position) and the end point (i.e. the short-term target position) at the same time. The new added path segments from the own-ship position are shown as blue lines, the new added path segments from the short-term target position are shown as red lines, and the intersecting path segments are shown as green lines.

After combining the greedy algorithm to remove redundant vertices from the obtained path planning 


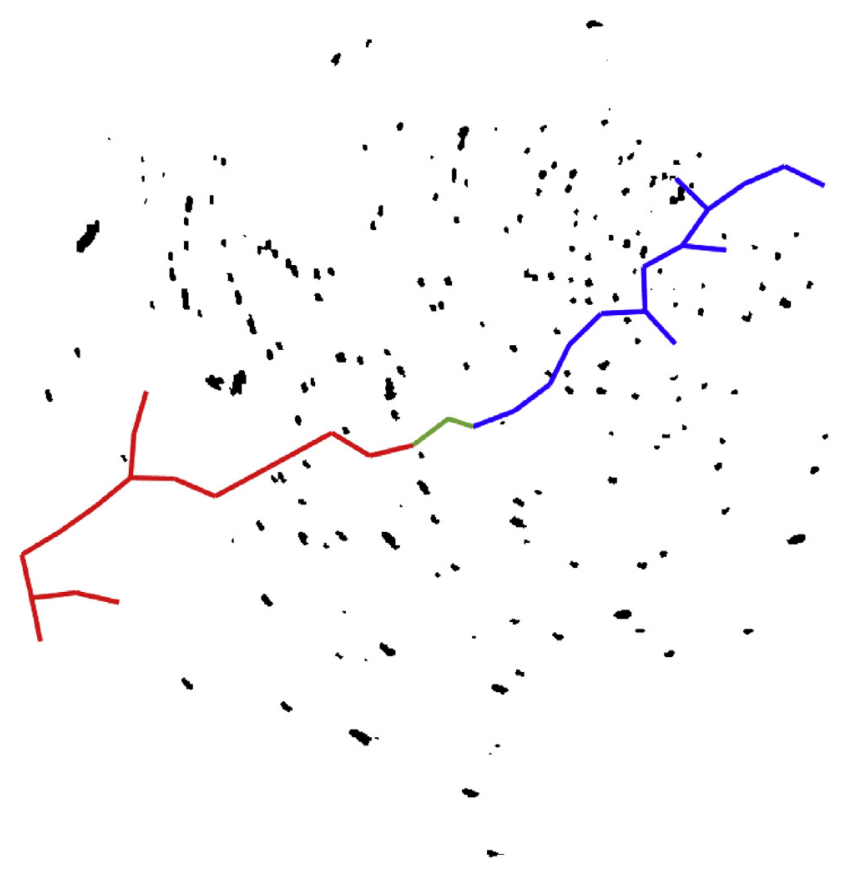

Fig. 8. Example of the operation of B-RRT algorithm.

scheme, as shown in Fig. 9, the original path planning scheme generated by the B-RRT algorithm is shown as a blue dashed line, with a total of 21 vertices. A new path planning scheme generated by combining the greedy algorithm is shown as a solid red line with six remaining vertices.

Under the premise that the original path planning scheme generated by the B-RRT algorithm is

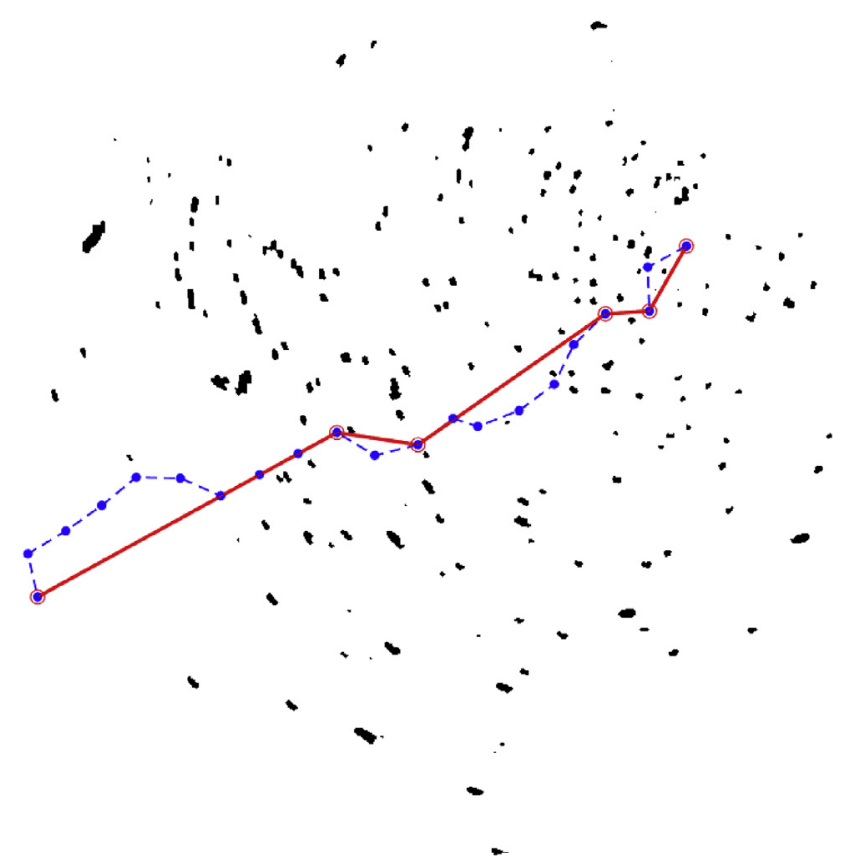

Fig. 9. Comparing the paths of B-RRT algorithm and greedy algorithm.

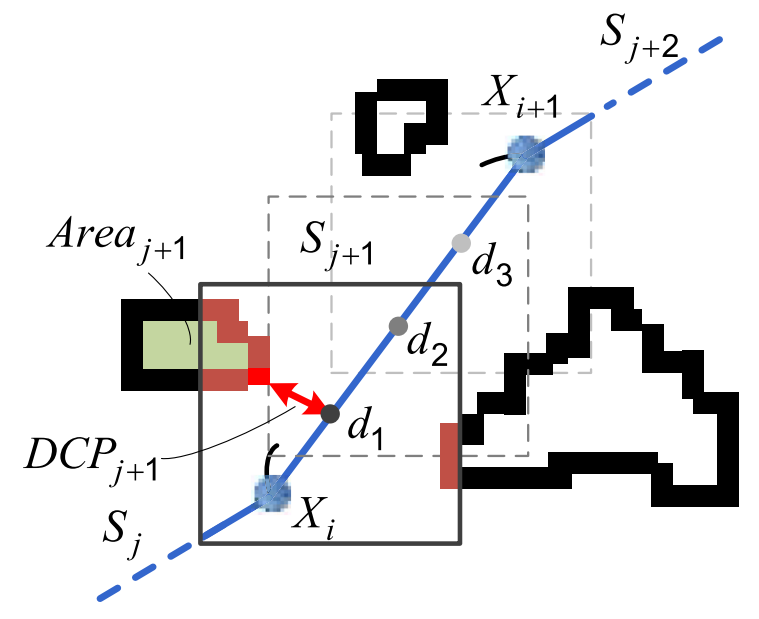

Fig. 10. Schematic diagram for the basic idea of PRI.

feasible, the redundant vertices can be removed by combining the greedy algorithm, thereby making the new path planning scheme simpler and more direct.

\subsection{Evaluation of path planning for ice navigation}

In order to evaluate the risk degree of the path planning for ice navigation on a radar image, based on the concept of radar sea ice risk index (RI) proposed in Chapter 3, this study proposes a path risk index (PRI) as shown in Fig. 10.

There is a path segment $S_{j+1}$ between the waypoint $X_{i}$ and $X_{i+1}$. On the path segment $S_{j+1}$, set a number of datum points (such as $d_{1}, d_{2}, d_{3}$, etc.) at fixed intervals (the distance of this study is set as 1 pixel), and establish a number of square test frames with each datum point as the center (the size of this study is set as $100 \times 100$ pixels). The exterior boundaries of each sea ice is marked within each square test frame (for example, the exterior boundaries of each sea ice within the $d_{1}$ square test frame are marked in red), and the distance calculated between the exterior boundaries of each sea ice and the datum point within the same square test frame to find the closest value and the closest point. By comparing these to the results obtained by other square test frames, the distance to the closest point within the scope of the $S_{j+1}$ path segment $\left(D C P_{j+1}\right)$ and the area of the sea ice covering the closest point within the scope of the $S_{j+1}$ path segment $\left(\right.$ Area $\left._{j+1}\right)$ can be obtained.

Accordingly, this study proposes a path risk index (PRI) by taking the distance to the closest point and the area of the sea ice covering the closest point as variables, as shown in Eq. (2): 


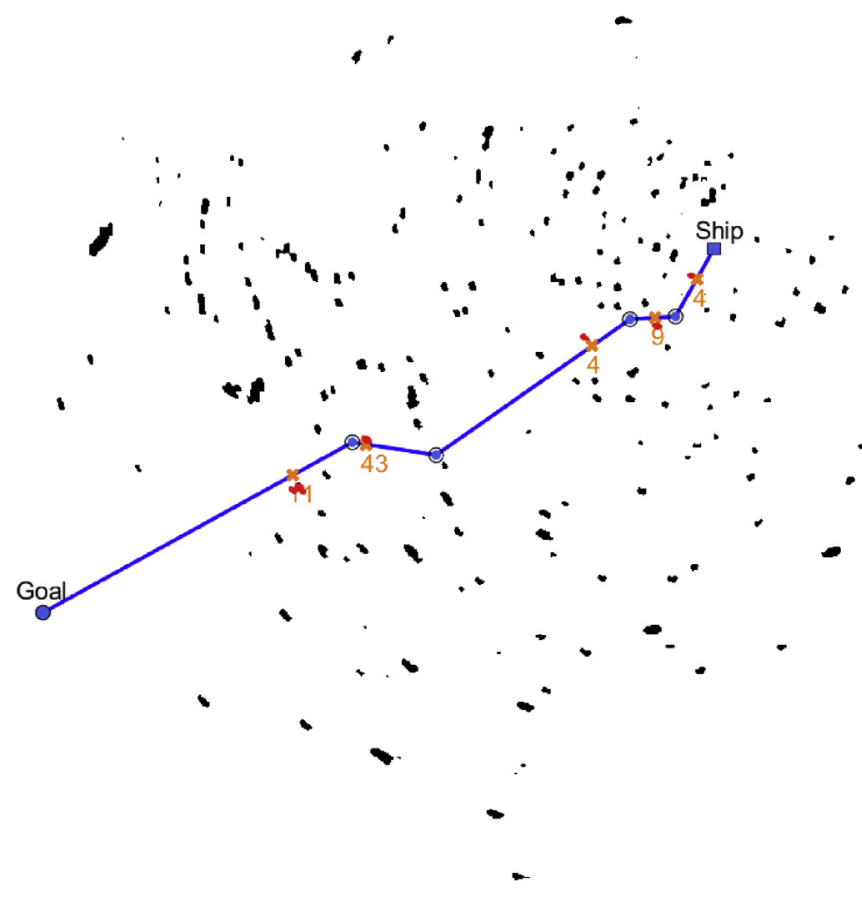

Fig. 11. Example of the calculation of PRI.

$P R I=\sum_{j=1}^{n} \frac{\text { Area }_{j}}{D P_{j}}$

in which $P R I$ is the risk index of the path planning on the radar image. Area $a_{j}$ is the area of the sea ice covering the closest point within the scope of the $j$ th path segment, in pixels. $D C P_{j}$ is the distance to the closest point within the scope of the $j$ th path segment, in pixels.

The area of the sea ice covering the closest point is directly proportional to the risk degree of the path; the larger the area of the sea ice covering the closest point, the higher the value of the PRI. The distance to the closest point is inversely proportional to the risk degree of the path; the closer the distance to the closest point, the higher the value of the PRI. The
PRI represents the relative risk degree of a given path; the higher the value of the PRI, the higher the risk degree of a given path. The PRI value of the entire path can be obtained by summing the PRI value of each path segment.

Taking the ice navigation scene reconstructed in Chapter 2 as an example, as shown in Fig. 11, the entire path is displayed on the radar image. On each path segment, the position closest to sea ice and its PRI value (rounded to integers) are respectively marked in orange crosses and numerics, and the

corresponding position of the sea ice is marked in red. In this example, the PRI value of the entire path is 71 (i.e., $4+9+4+43+11=71$ ).

\section{Example demonstration}

In order to demonstrate the effectiveness of the proposed path planning algorithms and the path risk index (PRI), we took the radar image captured from the radar playback video of the polar research vessel Xuelong on its Antarctic route at 6:53 on November 27, 2016 as an example. This study reconstructs the ice navigation scene and generates ten path planning schemes, as shown in Fig. 12.

\begin{tabular}{lllll}
\multicolumn{5}{l}{ Table } \\
\hline Path & Comparing the generated path planning schemes. & \\
& $\begin{array}{l}\text { Running } \\
\text { Time }(\mathrm{sec})\end{array}$ & $\begin{array}{l}\text { Number of } \\
\text { Vertices }\end{array}$ & Distance & PRI \\
\hline 1 & 3.9 & 5 & 851.0 & \\
2 & 4.4 & 5 & 927.0 & 34.3 \\
3 & 4.6 & 7 & 909.9 & 38.5 \\
4 & 7.1 & 5 & 887.2 & 21.9 \\
5 & 10.9 & 6 & 876.2 & 70.3 \\
6 & 10.7 & 6 & 870.2 & 43.8 \\
7 & 14.0 & 4 & 866.2 & 44.5 \\
8 & 5.3 & 4 & 874.7 & 23.1 \\
9 & 3.9 & 6 & 862.8 & 80.5 \\
10 & 8.6 & 6 & 909.4 & 68.8 \\
average & 7.3 & 5.4 & 883.4 & 46.0 \\
\hline
\end{tabular}

(4)

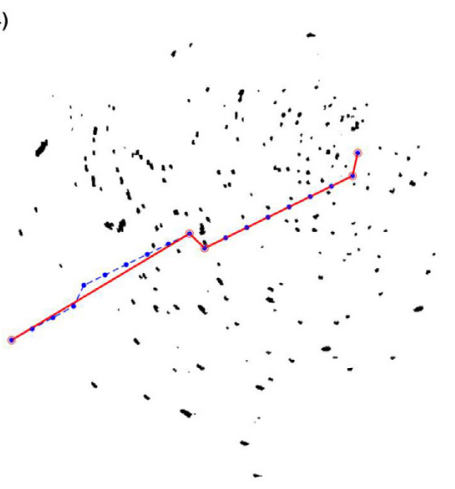

(1)

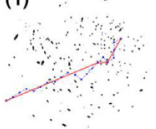

(5)

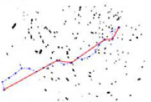

(8)

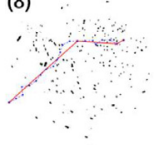

(2)

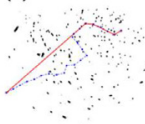

(6)

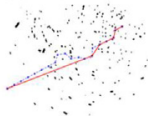

(9)

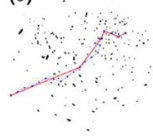

(3)

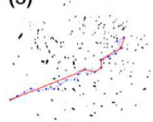

(7)

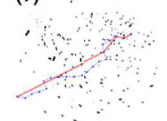

(10)

(10)

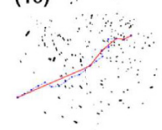

Fig. 12. Generating the ten path planning schemes. 

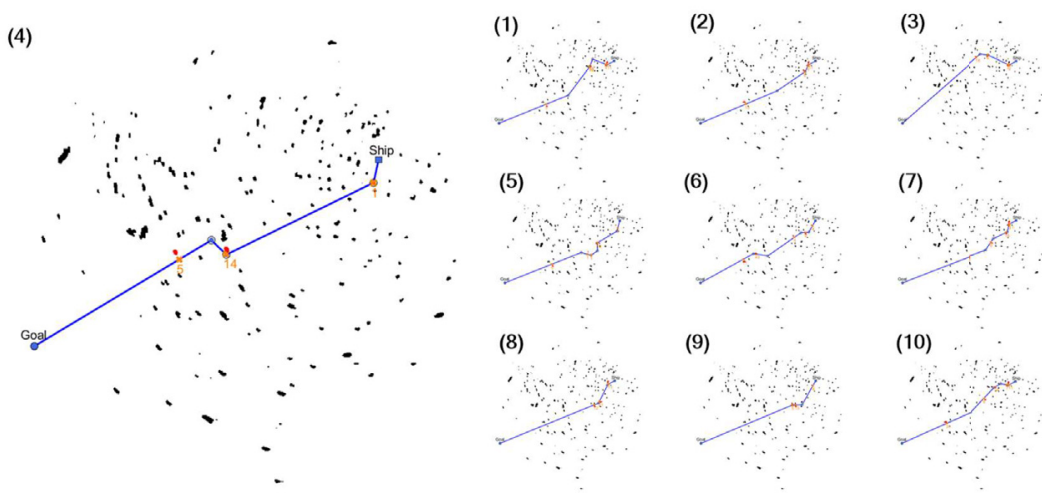

Fig. 13. Evaluating the ten path planning schemes.

As shown in Table 1, the generation time of each path is in the range of 3.9 seconds to 14 seconds, with an average time of 7.3 seconds. The demonstration results show that the path planning algorithm this paper proposes can generate ten path planning schemes within two minutes, which meets the requirements of computing time. Since the display range of the radar image is smaller, the navigation time of the small-scale path within this range is only about half an hour, and the computing time should not be too long. In addition, the number of vertices of each path is in the range of 4 to 7 , with an average of 5.4. The unnecessary vertices can be removed by combining the greedy algorithm. Furthermore, the distance of each path is in the range of 851.0 to 927.0 , with an average distance of 883.4. The demonstration results show that the difference in distance of each path is not significant, and the distance is not a primary consideration for choosing the path in ice navigation. Finally, the PRI value of each path is in the range of 21.9 to 80.5, with an average of 46.0. Compared with the distance, the PRI value directly affects the safety of navigation, and is a more important factor in ice navigation. The higher the value of the PRI, the higher the risk degree of a given path. In this example, path 4 , with the smallest PRI value, should be chosen as the relatively optimal path planning scheme.

As shown in Fig. 13, the recommended relatively optimal path planning scheme (path 4) and other path planning schemes are displayed on the radar image. In each path segment, the position closest to sea ice and its PRI value (rounded to integers) are respectively marked in orange cross and numerics.

\section{Conclusion}

In this study, we used a marine radar image to generate a small-scale path planning scheme for sea ice collision avoidance in ice navigation. At first, we used image recognition technology to recognize the own-ship position, short-term target position and unknown obstacle areas from the radar image, and to reconstruct the ice navigation scene. Next, we proposed the radar sea ice risk index to identify the risk degree of each sea ice area to the ship, and established a visualization function of sea ice warning on the radar image to assist the ice navigation. In addition, we used the bidirectional rapidly-exploring random tree (B-RRT) algorithm to generate the path planning scheme for sea ice collision avoidance, and then combined the greedy algorithm to remove redundant vertices of the path planning scheme to make the plan simpler and more direct, Finally, we proposed a path risk index to evaluate the given generated path planning schemes to choose the relatively optimal path planning scheme with lower risk. It is hoped that the results of this study will be helpful in path planning decisionmaking for ice navigation.

\section{Acknowledgments}

The research presented in this paper has been supported by the National Key R\&D Program of China (2019YFB1600605), Shanghai Science and Technology Innovation Action Plan (18DZ1206101), Young Teacher Training Program of Shanghai Municipal Education Commission (ZZHS18053), National Natural Science Foundation of China (51709167, 52071199), Shanghai Natural Science Foundation (18ZR1417100), Shanghai Pujiang Program (18PJD017), and Shanghai Shuguang Plan Project (15SG44).

\section{References}

[1] CCS. Guidelines for Polar Water Operational Manual, GD29-2016. 2016. China. (in Chinese).

[2] Choi M, Chung H, Yamaguchi H, De Silva LWA. Application of genetic algorithm to ship route optimization in ice navigation. In: Proceedings of the 22nd International Conference on Port and Ocean Engineering under Arctic Conditions (POAC), Espoo, Finland; 2013. 
[3] Choi M, Chung H, Yamaguchi H, Nagakawa K. Arctic sea route path planning based on an uncertain ice prediction model. Cold Reg Sci Technol 2015;109:61-9.

[4] Frederking R. A model for ship routing in ice. In: Proceedings of the 17th International Conference on Port and Ocean Engineering under Arctic Conditions (POAC); 2003. p. 467-76. Trondheim, Norway.

[5] Guinness RE, Saarimaki J, Ruotsalainen L, Kuusniemi H, Goerlandt F, Montewka J, et al. A Method for Ice-aware Maritime Route Optimization. In: Proceedings of IEEE/ION PLANS 2014, Monterey, CA; 2014. p. 1371-8.

[6] IMO. Adoption of the Revised Performance Standards for Radar Equipment, Resolution MSC.192(79). 2004. London.

[7] IMO. International Code for Ships Operating in Polar Waters (Polar Code), MEPC 68/21/Add.1. 2014. London.

[8] IMO. Guidelines for the Presentation of Navigation-related Symbols, Terms and Abbreviations, SN/Circ.243. 2014. London.

[9] IMO. Guidelines on Methodologies for Assessing Operational Capabilities and Limitations in Ice, MSC.1/Circ.1519. 2016. London.

[10] Kala R. Code for robot path planning using bidirectional rapidly-exploring random trees. Indian Institute of Information Technology Allahabad; 2014.

[11] Kaleschke L, Tian-Kunze X, Maaß N, Beitsch A, Wernecke A, Miernecki $M$, et al. SMOS sea ice product: Operational application and validation in the Barents Sea marginal ice zone. Remote Sens Environ 2016;180:264-73.

[12] Kotovirta V, Jalonen R, Axell L, Riska K, Berglund R. A system for route optimization in ice-covered waters. Cold Reg Sci Technol 2009;55(1):52-62.
[13] LaValle SM, Kuffner JJ. Randomized kinodynamic planning. Int J Robot Res 2001;20:378-400.

[14] LaValle SM. Planning Algorithms. 2006. Urbana-Champaign, Illinois.

[15] Lehtola V, Montewka J, Goerlandt F, Guinness R, Lensu M. Finding safe and efficient shipping routes in ice-covered waters: A framework and a model. Cold Reg Sci Technol 2019;165:1-14.

[16] Lehtola V, Montewka J, Salokannel J. Sea captains' views on automated ship route optimization in ice-covered waters. J Navigat 2020;73(2):364-83.

[17] Liu X, Sattar S, Li S. Towards an automatic ice navigation support system in the arctic sea. ISPRS Int J Geo-Info 2016;5: $36-47$.

[18] Montewka J, Goerlandt F, Lensu M, Kuuliala L, Guinness R. Toward a hybrid model of ship performance in ice suitable for route planning purpose. In: Proceedings of the Institution of Mechanical Engineers Part O: Journal of Risk and Reliability. 233; 2018. p. 18-34.

[19] Nam JH, Park IH, Lee HJ, Kwon MO, Choi KS, Seo YK. Simulation of optimal arctic routes using a numerical sea ice model based on an ice-coupled ocean circulation method. Int J Naval Architect Ocean Eng 2013;5:210-26.

[20] Reimer N. Ship trial for testing of an ice route optimization system. Copenhagen, Denmark: OTC Arctic Technology Conference; 2015.

[21] Takagi T, Tateyama K, Ishiyama T. Obstacle avoidance and path planning in ice sea using probabilistic roadmap method. In: Proceedings of the 22nd IAHR International Symposium on Ice; 2014. p. 510-7. Singapore. 